\title{
Metabolic state alteration of neural stem cells controls FAS-mediated apoptosis and neurogenesis
}

\author{
Zamawang F. Almemar ${ }^{1,2 *}$, Nicole H. Urban ${ }^{1}$, Leal K. Lauderbaugh ${ }^{1}$ \\ ${ }^{1}$ Department of Mechanical Engineering, University of Colorado at Colorado Springs, Colorado Springs, USA \\ ${ }^{2}$ Department of the Bioenergetics Institute, University of Colorado at Colorado Springs, Colorado Springs, USA \\ Email: ${ }^{*}$ zamawang.almemar@gmail.com
}

Received 27 June 2013; revised 27 July 2013; accepted 15 August 2013

Copyright (C) 2013 Zamawang F. Almemar et al. This is an open access article distributed under the Creative Commons Attribution License, which permits unrestricted use, distribution, and reproduction in any medium, provided the original work is properly cited.

\begin{abstract}
Metabolic stress causes an increased Fas-FasL (receptor-ligand pair) expression in Neural Stem Cells (NSCs) leading to Fas-induced apoptosis. In this study, we discuss the exposure of NSCs to different metabolic treatments that provoke cellular stress responses. We demonstrate that challenging cultured NSCs to ethanol (ETOH) increased cellular death via Fas-mediated apoptosis. Moreover, we establish that NSCs cultured under low lipid conditions, in which they were deprived of essential fatty acids, demonstrated increased cellular survival rates suggesting an increased ability for these lipid-starved cells to endure a stressed environment. This was further confirmed by exposing NSCs to low glucose levels and observing a decrease in percent death in low lipid NSCs. When stressed, NSCs have increased reactive oxygen levels and are susceptible to apoptosis. These findings indicate that under starved and stressed conditions, and in the presence of Fas Ligand (FasL), NSCs pursue fatty acid oxidation by burning fat as fuel. This may be the key to better understand the metabolic states of brain tumors and the characteristics of cancer.
\end{abstract}

Keywords: Neural Stem Cells; Apoptosis; Neurogenesis; Metabolic Stress

\section{INTRODUCTION}

The growing research interest in Neural Stem Cells (NSCs) is driven by the possible application of these multipotent cells as a therapeutic tool in neurodegene-rative disorders. NSCs exist in the developing central nervous system (CNS) and exhibit various metabolic states [1-3]. To create a fat deficiency in NSCs, a characteristic of viable tumor cells, C17s (derived from developing

${ }^{*}$ Corresponding author. mouse cerebellum) were deprived of all essential lipids, producing a low lipid (LL) cell line [4]. In this study, we evaluate the response of Normal and LL phenotype C17 cells to observe changes in their metabolic states and their reactions to stress. During neurogenesis, exposure to ethanol and low levels of glucose lead to limited NSC production [5-8]. They readily undergo apoptosis when environmental conditions are not ideal [9], such as altered metabolic states, hypoglycemic conditions, and ethanol-induced lipid-deprived environments [10-14]. Though a considerable amount of research has focused on the generation and development of neurons, the study of NSC health, growth and apoptosis in metabolic states which mimic disease states, is limited [15-18].

The literature indicates that metabolic interference leads to cellular stress conditions in NSCs resulting in various disease states, including cancer [19]. We studied the FasFasL expression in Normal and LL cells and observed that the stimulation of the Fas receptor displays both apoptotic and proliferative characteristics, affected by metabolic state or cellular stress, as in lipid/glucose deprived NSCs [20,21]. Furthermore, the Fas-FasL signaling has been shown to be crucial for the control of tumor suppression in NSCs [22]. NSCs express the Fas receptor (CD95) on their cell surface and together with its ligand (CD95L), Fas-FasL typically induces apoptosis [23,24], but here we show that it can also induce growth ${ }^{1}$. In this study, we establish that NSCs express varying responses and tend toward either cell death or cell proliferation when treated with different glucose levels, ETOH, or their combinations.

\section{MATERIALS AND METHODS}

\subsection{Cell Culture}

The C17.2 cell line adopted from the cerebellum of new-

${ }^{1}$ In this work, we assume that the predominant death mechanism is apoptosis, and this was confirmed in our Forward Scatter and Side Scatter data. 
born mice is capable of differentiating into neurons and is very sensitive to factors in their surrounding environments. The adherent C17.2 cell line was supplied by Dr. Evan Y. Snyder from Harvard Medical School, Boston, MA. C17.2 NSCs were cultured in two ways. Cells termed "Normal" were grown in Dulbecco's modified Eagle's medium (DMEM, GIBCO 11995) and supplemented with HEPES (GIBCO 1563-080), Pen/Strep, L-Glutamine, 10\% Fetal Bovine Serum (FBS, GIBCOBRL 10100139), and 5\% Normal Horse Serum (NHS, GIBCO 26050088). Cells termed "LL" (for low lipid) were cultured in low lipid media, also using DMEM, and supplemented with HEPES buffer, Pen/Strep, L-Glutamine, and 15\% delipidized Bovine Calf Serum (BCS, Pel-Freeze Biologicals 37117-5), based on the established procedure [25].

\subsection{C17.2 - Normal and Low Lipid Cell Preparation}

Cells were cultured in normal/unaltered FBS media for five-weeks. Once $2 \times 10^{6}$ to $5 \times 10^{6}$ cells per $\mathrm{mL}$ of medium were grown, the Normal cells were lysed and divided, with one half being cultured in normal media and the other half cultured in low lipid media. While the Normal cells were diluted to a factor of 1:200, the low lipid cells were diluted to 1:50, in their respective media. Due to differing growth rates, the dilution factors were selected to achieve a similar cell density (confluency) after the prescribed five weeks. Passages 13 - 18 were used for these studies.

\subsection{Cell Plating}

Normal cells were isolated and resuspended in their appropriate media (Normal or LL). $1 \times 10^{6}$ cells $/ \mathrm{mL}$ of each suspension was used for manual cell counting. The remaining cell suspension of each type was resuspended to $36 \times 10^{6}$ cells per $\mathrm{mL}$ of respective media. In the meantime, four 6-well plates were prepared and labeled accordingly, 2 for Normal and 2 for LL. Next, $6 \times 10^{6}$ cells per $\mathrm{mL}$ of the cell suspensions are added to the proper 6-well plates. In addition, $5.0 \mu \mathrm{g} / \mathrm{mL}$ of FasFc (R\&D Systems Inc. 435-FA) is added to each well. Finally, all four plates were incubated for 24 hours.

\subsection{Cell Line Treatments}

Normal and LL cells were treated with FasFc and incubated for 24 hours. The remaining wells were treated with $300 \mathrm{mM}$ of $95 \% \mathrm{ETOH}$ and $30 \mathrm{mM}$ Glc. Once again, the 6-well plates were incubated for 24 hours. For viability tests, two of the treated plates, one Normal and one LL, were used. Of the cell suspension, $100 \mu \mathrm{L}$ was injected into the flow cytometer for live/dead analysis; for manual cell counts, $10 \mu \mathrm{L}$ of the remaining cell suspensions was used for manual cell counting.

\subsection{Fas/Fas Ligand Staining}

Before treatment and staining with antibodies, cells were fixed using $1 \%$ paraformaldehyde (Sigma P-6148). Normal and LL NSCs were suspended with Accutase (Sigma A6964), washed three times with PBS and resuspended in PBS. Fixative was added to each Normal and LL tube. Both sets of tubes were incubated on ice for 20 minutes. For antibody treatment, Fas (PE conjugated hamster antiMouse CD95), IsoFas (PE anti-Armenian and Syrian hamster IgG cocktail), Fas Ligand (PE labeled anti-Mouse CD178), IsoFas Ligand (PE Rat IgG2bk isotype control) and Fc block (Purified Rat anti-Mouse CD16/ CD32) from BD BioSciences Pharmingen were prepared. The Normal and LL cells treated with fixative were washed three times and resuspended in PBS. To these cell suspensions, $0.5 \mathrm{mg} / \mathrm{mL}$ of Fc block was added to Normal and LL, and incubated on ice for 10 minutes. For antibody addition, $49.7 \times 10^{6}$ cells per $\mathrm{mL}$ of Normal and $11.22 \times 10^{6}$ cells per $\mathrm{mL}$ of LL were added to appropriate wells. This was followed by the addition of $0.02 \mu \mathrm{g} / \mathrm{mL}$ of the appropriate antibodies. For flow cytometry analyses, plates were incubated on ice for 20 minutes followed by resuspension and data collection. Five thousand events of each cell population were used in the flow cytometry analysis.

\section{RESULTS}

To test low-lipid and Normal phenotype expressions, the acidity of the lysosomal contents were examined, since lysosomes play a significant role in fatty acid storage, hence lipid-level indicator [25]. Normal cells have an adequate number of lysosomes, which are acidic organelles [26]. However, LL cells usually have a reduced number of lysosomes and are less acidic due to the reduced availability of fatty acid. Lysosensor (Molecular Probes-LysoSenso Green DND 189) indicates the pH levels of lysosomes. Likewise, Fas (Fas anti-Mouse PE) is a cell surface antibody that fluoresces when bound to surface Fas is present. The LL phenotype upregulates Fas expression compared to the Normal phenotype.

To determine the presence of the low lipid phenotype, Normal NSCs cultured in complete media as well as LL NSCs cultured in lipid-deficient media were first developed in culture flasks and tested for viability. After logphase cell growth, microscopic photographs of each subcell line were taken (Figures 1(A) and (B)) to provide a visual aid of the phenotypic variation between Normal and LL NSCs. As apparent from the photographs, Normal NSCs showed increased cell growth and their cell configuration elongated.

Fas anti-Mouse PE was used for determining the presence of the Fas receptor, and Lysosensor Fluorescent probe was used to measure lysosome $\mathrm{pH}$. NSCs were 

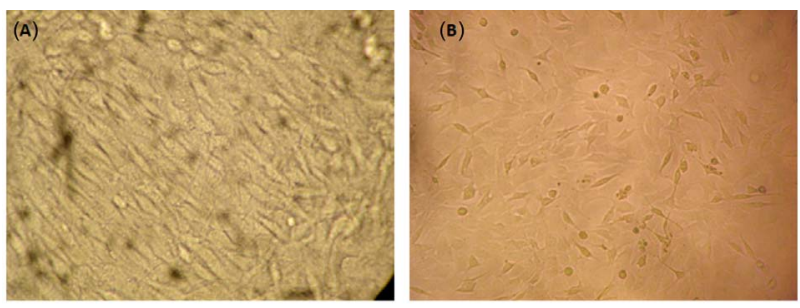

Figure 1. Normal and LL Neural Stem Cells (NSCs) display different phenotypes. (A) Microscopic photograph of Normal C17 NSCs show elongated shape and higher cell growth confluency. (B) Microscopic photograph of LL C17 NSCs illustrates a more spherical cell configuration, as well as reduced number of cells.

transferred to microplates and stained with corresponding antibodies. The Flow Cytometry data collection involved the analysis of 5000 events from each Normal and LL NSC suspension. The Flow Cytometry data (Figures 2(a) and (b)) displays an amplified level of Lysosensor (low $\mathrm{pH}$ ) in Normal NSCs, indicating healthy lysosomes. While the Flow Cytometry data indicates a higher expression of Fas on the low lipid NSC surface. Increased cell death was also observed in LL NSCs due to their lipid-deprived metabolic state.

The geometric mean value indicates the central tendency of the data [as well as identifies the intensity of fluorescence] and is used to analyze the logarithmicscaled data in Flow Cytometry [27]. LL NSCs exhibit greater Fas expression, indicated by the higher geometric mean value (Figure 2(a)). Although Normal NSCs display a higher peak, their fluorescence intensity is less ( $\mathrm{x}$ axis log scale) than the LL NSCs. As expected, the fluorescence data for the Lysosensor shows a higher geometric mean value and hence Lysosensor expression (low $\mathrm{pH}$ ) for Normal NSCs (Figure 2(b)). Since our fluorescence data displays higher fluorescence for Fas in LL than in Normal NSCs and higher Lysosensor fluorescence in Normal than in LL NSCs, we conclude that we have achieved Normal and LL phenotypes.

\subsection{Treatment of Normal and Low Lipid C17s Result in Apoptosis as Well as Neurogenesis}

Metabolic stresses, such as Ethanol, Glucose, and combination of these stressors contribute to altered response to Fas-mediated signaling. To determine the effects of glucose and ethanol induced stress as well as the role of metabolic state, Normal and LL NSCs were treated with $300 \mathrm{mM}$ ETOH, $30 \mathrm{mM}$ Glc, and combinations of these stressors. Additionally, FasFc was used as an experimental tool to block the Fas signaling pathway. NSCs were treated with FasFc to obstruct the Fas-receptor signaling an experimental treatment in our experimental design in combination with glucose and ETOH treatments. Disand inhibit apoptosis [28]. FasFc was primarily used as rupting the Fas-FasL pathway by way of FasFc protects NSCs from growth arrest or death.

The results are shown by the Flow data in Figure 3(a). The average data, obtained from four experimental repeats, indicates percent death to be $9.5 \%$ for Normal NSCs. Baseline Fas-mediated death observed in "No Add" treatments is $8.67 \%$ in Normal NSCs (solid bars) and the response to treatments are evaluated by comparing to the "No Add." Compared to "No Add" treatment, percent death increases upon the addition of ETOH to Normal NSCs, and with Glc added as an additional stressor to ETOH-treated NSCs, increased apoptosis is observed. At the same time, adding FasFc to ETOH-treated Normal NSCs gives an increase in cell death suggesting that blocking the Fas pathway does not protect NSCs from ETOH's effects. Moreover, the addition of Glc does not facilitate recovery for ETOH-affected NSCs, while similar percent deaths are observed when adding Glc to FasFc-treated Normal NSCs. Finally, adding Glc alone also acts as a stressor, displaying percent death results similar to the FasFc + Glc treatment.

To evaluate Fas-mediated death in LL NSCs, the same treatments were applied to cells of the LL phenotype (dashed bars). The percent death averages at $23.3 \%$ for LL NSCs for all treatments (compared to 9.5\% for Normal NSCs). The data for LL NSCs indicates an overall increase in susceptibility to apoptosis by displaying higher percentages of death. These results show that baseline Fas-mediated death observed in LL NSCs for the "No Add" treatments is $21.17 \%$. Detailed examination of LL NSC data demonstrates that adding ETOH increases the percent death from the baseline value. An increase in cell death was also revealed following treatment with ETOH and Glc combined. ETOH and Glc-treated LL NSCs experienced increased losses in number. Thus higher death rates suggest that once LL NSCs apoptose by ETOH, adding Glc acts as a death factor causing further apoptosis. However, the addition of FasFc alone results in a decrease in percent death compared to the baseline value. For further investigation of Fas-induced apoptosis in LL NSCs, FasFc was added to block the apoptotic pathway. FasFc addition to ETOH-treated LL NSCs increased percent death, once again supporting the fatal effects of NSC exposure to ETOH. In addition, adding FasFc to Glctreated LL NSCs also increased percent death, and the percent death result was similar to the Glc-only treatment.

By and large, the percent death increases with treatment compared with the data from the baseline "No dd" treatments and the absence of lipid increases the overall percent deaths. Furthermore, percent deaths in Normal treated NSCs show lower percentage of death, while LL NSCs exhibit higher death rates compared to their Normal and more healthy predecessors. In conclusion, 


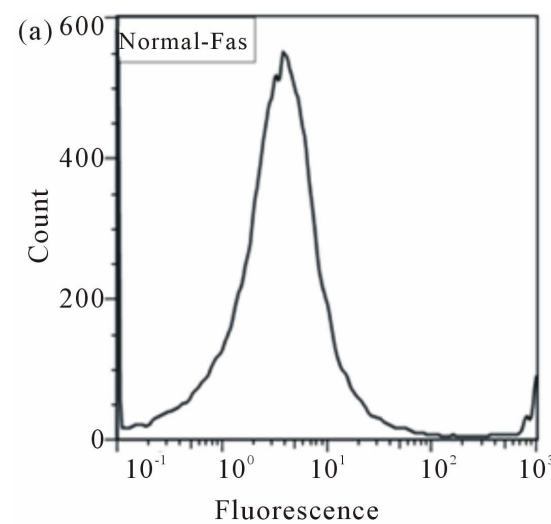

$\sum$ (FL2LOG)LFL2 Geom.Mean 2.91

(b)

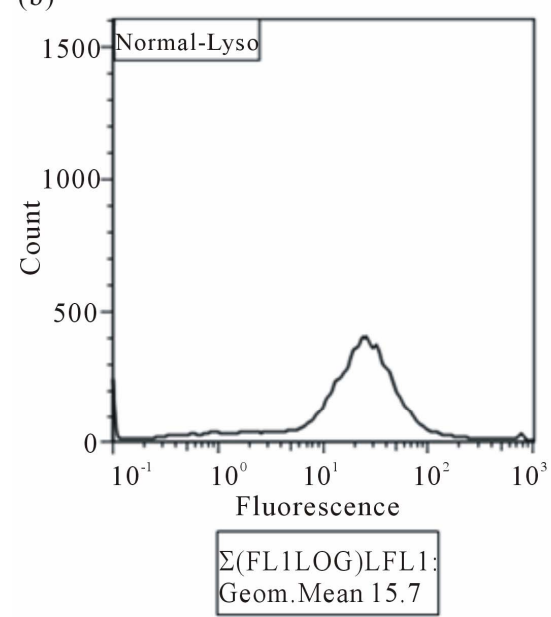

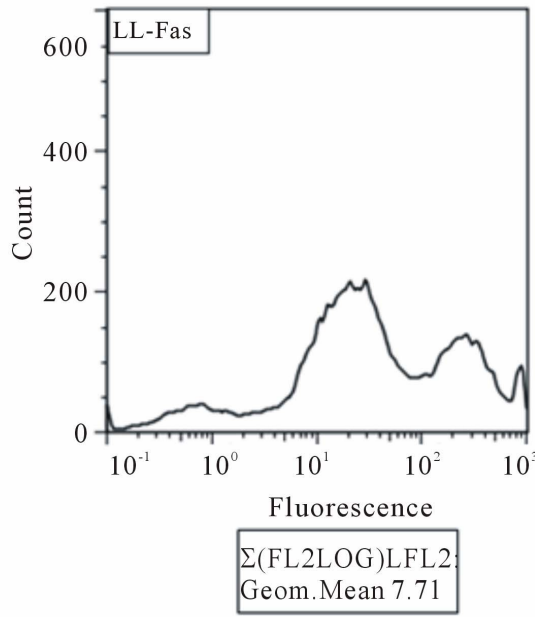

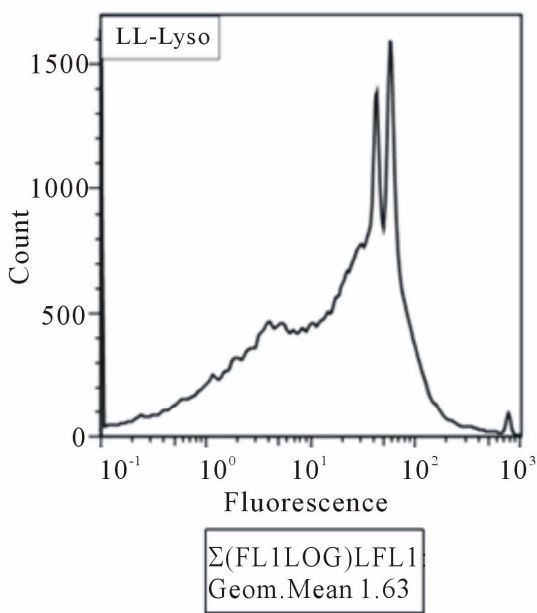

Figure 2. Flow Cytometry Data Illustrates Fas and Lysosensor Expressions by Normal and LL NSCs. (a) Flow cytometry and geometric mean analysis of Fas-treated Normal and LL NSCs indicates higher Fas expression in LL NSCs supporting the conclusion that LL NSCs show increased Fas-mediated death. (b) Flow cytometry and geometric mean analysis of Lysosensor-treated Normal and LL NSCs illustrates the conclusion that lack of essential lipids in LL NSCs and hence reduced fatty acid metabolism in the lysosome leads to decreased Lysosensor expression.

LL NSCs deprived of all lipids and essential fatty acids die Fas-mediated death due to the inadequate A culturing environment as well as ETOH and Glc treatments.

\subsection{Cell Count Data of Fas-Induced Death of NSCs Support Flow Cytometry Data}

To validate the Flow Cytometry data, manual cell counts of each treated and untreated Normal and LL NSCs were obtained [29]. The Cell Count data confirms the same general trends achieved by the Flow Cytometer (collected via Coulter Elite Epics or Excel Flow Cytometer and analyzed via FlowJo version 7.0). Evaluating forward scatter and side scatter of 5000 events of each sample population provides a baseline interpretation of the data. Fas-mediated cell death is observed from the ETOH treatment in Normal NSCs (Figures 3(a) and (b)). The Cell Count data confirms that the addition of ETOH causes the highest amount of cell death in Normal NSCs and that ETOH induced death is observed regardless of whether ETOH was added independently or in combination with FasFc or Glc (Figures 3(b)). The following results again confirm our additional conclusion that FasFc, used to block the Fas pathway, protects NSCs from death when added to untreated Normal NSCs.

Further inspection of the preceding data demonstrates that the same trends are observed in LL from the Cell Count data as compared to the Flow data. As seen from both the Cell Count and Flow data, FasFc addition to ETOH-treated LL increases the percent death, again supporting the conclusion that ETOH-induced death is not Fas-induced. 

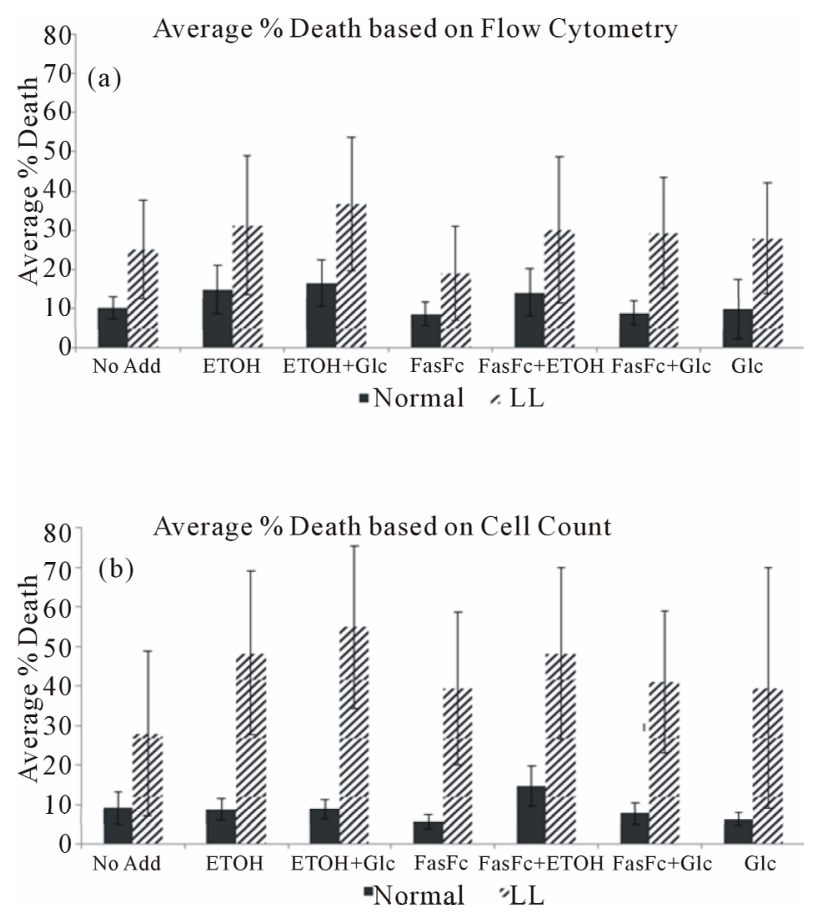

Figure 3. Cell Count Data Follows Similar Trends from Flow Cytometry Data for All Treatments. (a) Average percent deaths from Flow Cytometry data illustrate highest death rates in the presence of ETOH. (b) Average percent death from manual cell count for same treatments as shown in (a). Error bars represent standard error of the mean (SEM) based on four experimental trials.

We further examined cellular proliferation using cell count results and next we present the data supporting the survival rates of Normal and LL NSCs. It is noteworthy that average percent deaths resulting from analysis of Flow Cytometry and cellularity data provide comparable trends.

\subsection{Cellularity Data Validate Death Trends}

To further analyze Fas-mediated apoptosis and Fas-mediated proliferation, cell survival rates (cellularity) of Normal and LL NSCs were examined (Figures 4). The data show that compared to the "No Add" baseline data, decreased survival rates were observed in treatments with ETOH and ETOH and Glc combination in Normal NSCs. However, adding Glc to ETOH-treated LL NSCs reduces ETOH-induced death. Adding ETOH decreases cellularity in both cell lines. Blocking the Fas pathway with the addition of FasFc also decreases cellularity in both cell lines. An interesting observation is made when the apoptotic pathway is blocked and NSCs are induced by ETOH. In Normal NSCs, ETOH reduces cell survival rates despite the addition of FasFc, however in LL NSCs upon the addition of ETOH in combination with FasFc, higher survival rates are observed, compared to ETOH- only and FasFc-only treatments. The same trend is true in Normal and LL NSCs when Glc is added and the Fas pathway is blocked. Cellularity decreases in Normal NSCs whereas somewhat increases in LL NSCs, compared to the FasFc-only treatment. Furthermore, with the apoptotic pathway blocked, Glc-treated Normal NSCs display higher survival rates than ETOH-treated NSCs, while LL NSCs demonstrate decreased cellularity than their ETOHtreated counterparts. Cellularity is further decreased in LL NSCs in the Glc-only treatment; however, expected results are obtained in Normal NSCs with increased cellularity upon the addition of Glc.

\subsection{Low Lipid NSCs Survive Low Glucose Levels}

NSCs were treated with low Glc levels. Glucose deprivation decreases the number of Normal NSCs, while increasing the percent death (Figure 5). As shown by the cellularity data, LL NSCs also decrease in number, but interestingly their percent death decreases as well. Whether changes of death rates or cellularity are analyzed, they both follow the same statistical trends. This suggests the utilization of a unique survival mechanism by the LL phenotype when sources of lipid and glucose are scarce, which could be speculated as fatty acid oxidation.

\section{DISCUSSION}

\subsection{Metabolic Stress States and Survival Mechanisms of LL NSCs}

To investigate the effect of different treatments applied to both Normal and LL NSCs, the death rate variations between the two sub-cell lines are studied. Average percent death increases by about $14 \%$ when examining LL compared to Normal NSCs for all treatments. We conclude that LL NSCs are more sensitive to apoptotic effects of the Fas-FasL death receptor-ligand pair, therefore more

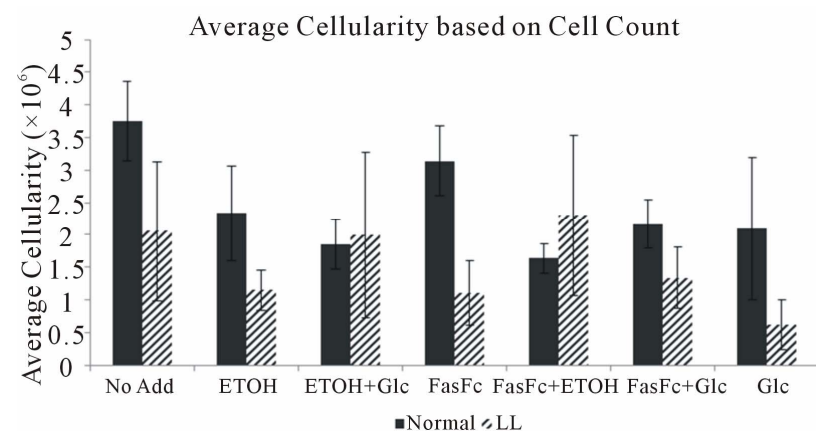

Figure 4. Cellularity Analysis of Normal and LL NSCs Indicate Dissimilar Outcomes upon Glc Addition. Average total cellularity data of Normal and LL NSCs generally follow the same trends upon treatments, except in FasFc + ETOH and Glc treatments. 

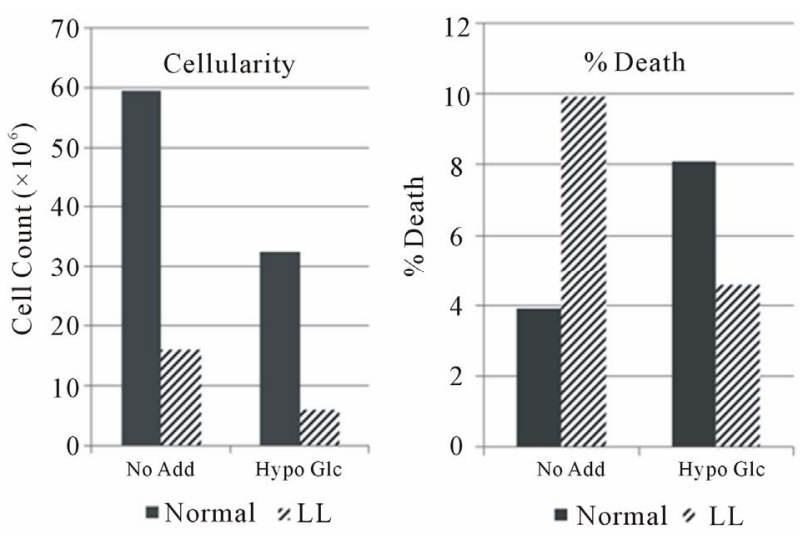

Figure 5. Cellularity and Percent Death Analysis of Normal and LL NSCs Suggest Survival Mechanism used by LL NSCs. Normal NSCs demonstrate decreased cellularity and increased percent death under conditions of low glucose levels. While, LL NSCs show decreased cellularity and decreased percent death, illustrating this phenotype's capability of survival under severe conditions.

death is observed in LL NSCs as a result of Fas induction. Interestingly, one of the indicators of the LL phenotype is an upregulation of the Fas receptor. This is exclusively caused by depriving the cell line from lipids. LL NSCs recover only by a small amount when treated with Glc suggesting that Glc causes Fas activation by binding to the Fas receptors and altering the NSC's fate from Fasinduced apoptosis to Fas-mediated proliferation. In LL NSCs, Glc upregulates the Fas-FasL binding pathway and allows more receptors to bind.

We have demonstrated that treatment of Normal NSCs with ETOH results in increased death rates. However, LL NSCs survive better in these stressed environments. In fact, neurogenesis is observed in these LL NSCs upon the addition of the ETOH + Glc and FasFc + ETOH treatments. It is reasonable to conclude that under metabolic stress conditions, such as under conditions of limiting glucose supplies, these NSCs undergo fatty acid oxidation as a source of carbon [4]. Furthermore, FasL is provided only in presence of lipid during fatty acid oxidation by burning fat as fuel, and fatty acid oxidation by LL NSCs leads to FasL dependency [19]. This finding establishes a link between the cellular metabolic strategy used by LL NSCs and demonstrates the potential to further study these LL NSCs to understand other diseases that involve Glc.

\subsection{Metabolic Stresses, Apoptosis and Neurogenesis in NSCs}

The surface receptor Fas (CD95) has long been identified as the primary pathway for apoptosis (programmed cell death). However, several researchers have documented cell proliferation when Fas is triggered. Fas-activated
NSCs can undergo either apoptosis or cell proliferation [23]. Our data indicate that this bifurcation is affected by their phenotypic state and type of treatment administered. Normal and LL C17s undergo Fas-mediated apoptosis, indicated by the cellularity data. These results introduce a novel insight into Fas-FasL-induced apoptosis in C17 NSCs. In this research, the effect of metabolic stresses, which mimic known disease states was investigated in NSCs. The highest death rates were detected when ETOH was administered as one of the treatments to the developing Normal and LL NSCs. FASD results in neuronal loss due to ethanol-induced damage [7]. Our results coincide with these findings and show increased apoptotic activity in Normal and LL NSCs exposed to ETOH.

We have identified that FasFc protects NSCs from growth arrest (death) and that administering FasFc to NSCs exposed to ETOH protects the NSCs by blocking the Fas-FasL receptors, decreasing Fas-dependent death. At the same time, increased percent deaths are observed with ETOH-treated NSCs. We conclude that ETOH-induced death is Fas-independent since death is observed in both Normal and LL NSCs regardless of whether or not the apoptotic pathway is blocked. Likewise, we conclude that Glc-dependent growth is also Fas-independent since increased numbers of cells are observed in LL NSCs while it inhibits growth in Normal NSCs when the apoptotic pathway is blocked. This variation between Glc-treated Normal and LL NSCs indicates that LL NSCs use this Glc as fuel to drive Fas-mediated cell proliferation under lipid-deprived conditions by means of fatty acid oxidation. However, adding Glc to LL NSCs when the apoptotic pathway is not blocked acts as an added stressor, decreasing cellularity. Collectively, these observations demonstrate that the Fas-mediated pathway can lead to apoptosis and proliferation, which is affected by the metabolic state of NSCs (i.e. normal conditions or stressed and lipid-deprived environments).

\subsection{Fas and Metabolic Stressors}

We have demonstrated that NSCs treated with ETOH lead to death and that death is not Fas-mediated. We have further identified that Glc-dependent growth in NSCs is also not Fas-mediated. However, under metabolic stress conditions and in NSCs that are lipid-deprived, treatment with Glc leads to Fas activation, altering the apoptotic pathway to lead to cellular proliferation instead of death. Our studies of ETOH, Glc, and FasFc treatments of NSCs advocate our conclusions that ETOH-induced death is Fas-FasL independent and that Glc-dependent growth is also Fas-FasL independent. Unraveling the factors affecting Fas activation in LL NSCs could enable the use of Fas to further our understanding of cancer. 


\section{ACKNOWLEDGEMENTS}

The authors would like to acknowledge the technical support and guidance of Dr. M. K. Newell-Rogers, and for services provided by the University of Colorado at Colorado Springs Bioenergetics Laboratory facility. The authors have no conflicts of interest to declare.

\section{REFERENCES}

[1] Reynolds, B.A. and Weiss, S. (1992) Generation of neurons and astrocytes from isolated cells of the adult mammalian central nervous system. Science, 255, 1707-1710. http://dx.doi.org/10.1126/science.1553558

[2] Gage, F.H., Coates, P.W., Palmer, T.D., Kuhn, H.G., Fisher, L.J., Suhonen, J.O., Peterson, D.A., Suhr, S.T. and Ray, J. (1995) Survival and differentiation of adult neuronal progenitor cells tranplanted to the adult brain. Proceedings of the National Academy of Sciences, 92, 1187911883. http://dx.doi.org/10.1073/pnas.92.25.11879

[3] Taupin, P. and Gage, F.H. (2002) Adult neurogenesis and neural stem cells of the central nervous system in mammals. Journal of Neuroscience Research, 69, 745-749. http://dx.doi.org/10.1002/jnr.10378

[4] Harper, M., Antoniou, A., Villalobos-Menuey, E., Russo, A., Trauger, R., Vendemelio, M., George, A., Bartholomew, R., Carlo, D., Shaikh, A., Kupperman, J., Newell, E., Bespalov, I., Wallace, S., Liu, Y., Rogers, J., Gibbs, G., Leahy, J., Camley, R., Melamede, R. and Newell, K. (2002) Characterization of a novel metabolic strategy used by drug-resistant tumor cells. The FASEB Journal, 16, 1550-1557. http://dx.doi.org/10.1096/fj.02-0541com

[5] Vangipuram, S.D. and Lyman, W.D. (2010) Ethanol alters cell fate of fetal human brain-derived stem and progenitor cells. Alcoholism: Clinical and Experimental Research, 34, 1574-1583.

http://dx.doi.org/10.1111/j.1530-0277.2010.01242.x

[6] Kim, K.C., Go, H.S., Bak, H.R., Choi, C.S., Choi, I., Kim, P., Han, S., Han, S.M., Shin, C.Y. and Ko, K.H. (2010) Prenatal exposure of ethanol induces increased glutamatergic neuronal differentiation of neural progenitor cells. Journal of Biomedical Science, 17, 1-9. http://dx.doi.org/10.1186/1423-0127-17-85

[7] Prock, T.L. and Miranda, R.C. (2007) Embryonic cerebral cortical progenitors are resistant to apoptosis, but increase expression of suicide receptor DISC-complex genes and suppress autophagy following ethanol exposure. Alcoholism: Clinical and Experimental Research, 31, 694703.

[8] Dikranian, K., Quin, Y., Labruyere, J., Nemmers, B. and Olney, J.W. (2005) Ethanol-induced neuroapoptosis in the developing rodent cerebellum and related brain stem structures. Developmental Brain Research, 155, 1-13. http://dx.doi.org/10.1016/j.devbrainres.2004.11.005

[9] Ceccatelli, S., Tamm, C., Sleeper, E. and Orrenius, S. (2004) Neural stem cells and cell death. Toxicology Letter, 149, 59-66. http://dx.doi.org/10.1016/j.toxlet.2003.12.060

[10] Moreno-Sanchez, R., Saavedra, E., Rodriguez-Enriquez, S., Gallardo-Perez, J.C., Quezada, H. and Westerhoff, H.V. (2010) Metabolic control analysis indicates a change of strategy in the treatment of cancer. Mitochondrion, 10, 626-639. http://dx.doi.org/10.1016/j.mito.2010.06.002

[11] Cavaliere, F., D’Ambrosi, N., Sancesario, G., Bernardi, G. and Volonte, C. (2001) Hypoglycaemia-induced cell death: Features of neuroprotection by the P2 receptor antagonist basilen blue. Neurochemistry International, 38, 199-207. http://dx.doi.org/10.1016/S0197-0186(00)00087-5

[12] Auer, R.N. (1986) Progress review: Hypoglycemic brain damage. Stroke, 17, 699-708. http://dx.doi.org/10.1161/01.STR.17.4.699

[13] Mitchell, J.J., Paiva, M., Moore, D.B., Walker, D.W. and Heaton, M.B. (1998) A comparative study of ethanol, hypoglycemia, hypoxia and neurotrophic factor interactions with fetal rat hippocampal neurons: A multi-factor in vitro model for developmental ethanol effects. Developmental Brain Research, 105, 241-250. http://dx.doi.org/10.1016/S0165-3806(97)00182-X

[14] Suh, S.W., Hamby, A.M., Gum, E.T., Shin, B.S., Won, S.J., Sheline, C.T., Chan, P.H. and Swanson, R.A. (2008) Sequential release of nitric oxide, zinc and superoxide in hypoglycemic neuronal death. Journal of Cerebral Blood Flow and Metabolism, 28, 1697-1706.

http://dx.doi.org/10.1038/jcbfm.2008.61

[15] Geuna, S., Borriione, P., Fornaro, M. and Giacobini-Robecchi, M.G. (2001) Adult stem cells and neurogenesis: Historical roots and state of the art. The Anatomical Record, 265, 132-141. http://dx.doi.org/10.1002/ar.1135

[16] Cao, Q., Benton, R.L. and Wittemore, S.R. (2002) Stem cell repair of central nervous system injury. Journal of Neuroscience Research, 68, 501-510. http://dx.doi.org/10.1002/jnr.10240

[17] Horie, N., Moriya, T., Mitome, M., Kitagawa, N., Nagata, I. and Shinohara, K. (2004) Lowered glucose suppressed the proliferation and increased the differentiation of murine neural stem cells in vitro. FEBS Letters, 571, 37-242. http://dx.doi.org/10.1016/j.febslet.2004.06.085

[18] Wu, Z., Huang, K., Yu, J. H., Le, T., Namihira, M., Liu, Y., Zhang, J., Xue, Z., Cheng, L. and Fan, G. (2012) Dnmt3a regulates both proliferation and differentiation of mouse neural stem cells. Journal of Neuroscience Research, 90, 1883-1891.

[19] Newell, M.K., Melamede, R., Villalobos-Menuey, E., Swartzendruber, D., Trauger, R., Camley, R.E. and Crisp, W. (2004) The effects of chemotherapeutics on cellular metabolism and consequent immune recognition. Journal of Immune Based Therapies and Vaccines, 2, 1-6. http://dx.doi.org/10.1186/1476-8518-2-3

[20] Zimmermann, K.C., Bonzon, C. and Green, D.R. (2001) The machinery of programmed cell death. Pharmacology and Therapeutics, 92, 57-70. http://dx.doi.org/10.1016/S0163-7258(01)00159-0

[21] Strasser, A., Jost, P.J. and Nagata, S. (2009) The many roles of FAS receptor signaling in the immune system. Immunity, 30, 180-192. http://dx.doi.org/10.1016/j.immuni.2009.01.001

[22] Mahmood, Z. and Shukla, Y. (2010) Death receptors: Targets for cancer therapy. Experimental Cell Research, 6, 887-899. http://dx.doi.org/10.1016/j.yexcr.2009.12.011 
[23] Corsini, N.S., Sancho-Martinez, I., Laudenklos, S., Glagow, D., Kumar, S., Letellier, E., Koch, P., Teodorczyk, M., Kleber, S., Klussmann, S., Wiestler B., Brustle O., Mueller, W., Gieffers, C., Hill, O., Thiemann, M., Seedorf, M., Gretz, N., Sprengel, R., Celikel, T. and Martin-Villalba, A. (2009) The death receptor CD95 activates adult neural stem cells for working memory formation and brain repair. Cell Stem Cell, 5, 178-190. http://dx.doi.org/10.1016/j.stem.2009.05.004

[24] Knight, J.C., Scharf, E.L. and Draayer, Y. (2010) Fas activation increases neural progenitor cell survival. Journal of Neuroscience Research, 88, 746-757.

[25] Schweitzer, S.C., Reding, A.M., Patton, H.M., Sullivan, T.P., Stubbs, C.E., Villalobos-Manuey, E., Huber, S.A. and Newell, M.K. (2006) Endogenous versus exogenous fatty acid availability affects lysosomal acidity and MHC class II expression. Journal of Lipid Research, 47, 2525-2537. http://dx.doi.org/10.1194/jlr.M600329-JLR200
[26] Nixon, R., Mathews, P.M. and Cataldo, A.M. (2001) The neuronal endosomal-lysosomal system in alzheimer's disease. Journal of Alzheimer's Disease, 3, 97-107.

[27] Shapiro, H.M. (2003) Practical flow cytometry. John Wiley \& Sons Inc., Hoboken, 225-256.

[28] Pajusto, M., Tarkkanen, J. and Mattila, P.S. (2005) Human primary adenotonsillar naïve phenotype CD45RA+ CD4+ T lymphocytes undergo apoptosis upon stimulation with a high concentration of CD3 antibody. Scaninavian Journal of Immunology, 62, 546-551. http://dx.doi.org/10.1111/j.1365-3083.2005.01697.x

[29] Ryder, E.F., Snyder, E.Y. and Cepko, C.L. (1990) Establishment and characterization of mulipotent neural cell lines using retrovirus vector-mediated oncogene transfer. Journal of Neurobiology, 21, 356-375. http://dx.doi.org/10.1002/neu.480210209 